\title{
Fortificação do leite fluido na prevenção e tratamento da anemia carencial ferropriva em crianças menores de 4 anos
}

\section{Fortification of fluid milk for the prevention and treatment of iron defficiency anemia in children under 4 years of age}

\author{
Marco A. A. Torres, Neil F. Lobo, Kazue Sato e Suzana de Souza Queiroz \\ Divisão do Grupo de Pesquisas do Núcleo de Nutrição do Centro de Referência de Saúde \\ da Mulher, Nutrição Alimentação e Desenvolvimento Infantil da Secretaria de Saúde de São Paulo. \\ São Paulo, SP - Brasil (M.A.A.T., K.S.); Departamento de Medicina Preventiva da Universidade \\ Federal de São Paulo. São Paulo, SP - Brasil (N.F.L.); D epartamento de Pediatria da Faculdade de \\ Medicina de Botucatu. Universidade Júlio de Mesquita Filho. Botucatu, SP - Brasil (S.S.Q.)
}

\begin{abstract}
Resumo
Observou-se a eficácia da fortificação do leite fluido com $3 \mathrm{mg}$ de ferro aminoácido quelato no combate à carência de ferro em crianças menores de quatro anos. Foram acompanhadas 269 crianças que receberam, durante 12 meses, um litro de leite fortificado por dia, e que foram avaliadas a cada 6 meses de acompanhamento. Antes de se iniciar a intervenção, a anemia estava presente em $62,3 \%$ das crianças. Após 6 meses, este percentual reduziu-se a $41,8 \%$ e, ao final de um ano, a $26,4 \%$. As maiores reduções foram detectadas nas faixas etárias de 12 a 23 meses e em menores de um ano. Das crianças que apresentavam hemoglobinas iniciais inferiores a 9,5 g/dl, 59,3\% recuperaramse da anemia ao final de um ano de acompanhamento. Naquelas com hemoglobinas iniciais entre 9,5 e 10,9 g/dl, o percentual de recuperação da anemia foi de $66,7 \%$. Encontrou-se, ainda, melhores evoluções hematológicas em crianças que ingeriam quantidades superiores a $750 \mathrm{ml} /$ dia de leite fortificado, pertencentes a famílias que não dividiam o suplemento recebido com outros membros e naquelas com apenas uma criança com menos de 5 anos no núcleo familiar. Concluiu-se pela viabilidade e eficácia da fortificação do leite fluido como medida de intervenção no combate à carência de ferro em pré-escolares.
\end{abstract}

Anemia ferropriva, prevenção \& controle. Alimentos fortificados, utilização. Suplementação alimentar.

\begin{abstract}
The effectiveness of the use of chelate aminoacid iron fortified fluid milk in the treatment of iron deficiency in children under four years of age was studied. The 269 children included in this trial received 1 liter/day offluid milkfortified with $3 \mathrm{mg}$ of chelate aminoacid iron and were evaluated at six monthly intervals. At the beginning of the study $62.3 \%$ of the children presented anemia. After 6 months, this percentage had decreased to $41.8 \%$ and at the end of one year to $26.4 \%$. The greatest decreases occurred in the groups comprising
\end{abstract}

Correspondência para/Correspondence to: Marco Antonio de Almeida Torres - Rua Dr. Augusto de Miranda, 1297 - 05026-001 São Paulo, SP Brasil. Fax:(011) 873.3911

Edição subvencionada pela FAPESP. Processo 95/2290-6.

Recebido em 1.11.1995. Aprovado em 27.2.1996. 
the subjects who were of 12 to 23 months of age and those under one year of age. Among the children who presented initial hemoglobin levels under $9.5 \mathrm{~g} /$ $d l, 59.3 \%$ were free of anemia after one year of follow-up. Of those presenting initial hemoglobin levels between 9.5 and $10.9 \mathrm{~g} / \mathrm{dl}$, 66.7\% recovered from their anemia. There was also greater hematological improvement in the children that ingested over $750 \mathrm{ml} /$ day of fortified milk in those families that did not share the supply of supplement among their other members and in those families that had only one child under five years of age. These findings led to the conclusion that the fortification of fluid milk is a viable and effective method for the treatment of iron deficiency in pre-school children.

Anemia, iron-deficiency, prevention and control. Food, fortified, utilization. Supplemantary feeding.

\section{INTRODUÇÃO}

O combate à anemia carencial ferropriva, devido a sua elevada prevalência e conseqüências sobre o crescimento, desenvolvimento e mortalidade da população infantil, principalmente em menores de 2 $\operatorname{anos}^{2,7,9,15,18,22}$,é, hoje, uma das prioridades para os profissionais responsáveis pelo planejamento de Programas de Nutrição em Saúde Pública ${ }^{5}{ }^{12}$. Esta afirmação encontra respaldo político no compromisso assumido pelo Brasil e mais 159 países participantes da Conferência Internacional em Nutrição da FAO/OMS, em Roma, de reduzir a prevalência desta patologia até o ano $2.000^{6}$.

A busca de alternativas para o combate à carência de ferro, que melhor se adaptem às condições de cada região, tem sido motivo de várias publicações ${ }^{1,3}$. Entre elas, a fortificação dos alimentos que compõem a dieta básica da população-alvo tem demonstrado excelentes resultados $8,10,11,17$.

A partir da constatação de que $59,1 \%$ dos menores de 24 meses que freqüentam as Unidades Básicas de Saúde (UBS), em todo o Estado de São Paulo apresentam anemia - níveis de hemoglobina inferiores a $11,0 \mathrm{~g} / \mathrm{dl}$ e $25,1 \%$ anemia grave - e hemoglobinas inferiores a $9,5 \mathrm{~g} / \mathrm{dl}^{19}$, a Secretaria de Estado da Saúde de São Paulo vem, desde 1989, buscando e avaliando alternativas para o controle dessa carência nutricional no Estado, e detectou que:

- A profilaxia medicamentosa, apesar de eficiente do ponto de vista da recuperação das condições hematológicas das crianças avaliadas, apresentou sérios entraves operacionais ${ }^{13,20}$.

- A experiência com o enriquecimento do leite em pó mostrou que a utilização de pequenas doses diárias de ferro é capaz de prevenir e recuperar a anemia carencial ferropriva em menores de 2 anos, num curto espaço de tempo ${ }^{21}$.

Em 1984, o Programa de Suplementação Alimentar da citada Secretaria substituiu a preconização do leite em pó e incorporou a distribuição do leite fluido para crianças menores de 5 anos em todo o interior do Estado, e manteve a distribuição do leite em pó na região da Grande São Paulo. Em 1992, iniciou a distribuição do leite em pó fortificado com ferro e vitaminas $\mathrm{A}, \mathrm{C}$ e D para menores de 24 meses de idade, atendidos nas UBS da Grande São Paulo.

O surgimento de uma tecnologia que permitiu a fortificação do leite fluido veio possibilitar a manutenção do programa no interior do Estado, bem como incorporar o combate à anemia carencial ferropriva em suas metas.

O presente estudo foi realizado com o objetivo de verificar a viabilidade e a eficácia da fortificação do leite fluido com ferro aminoácido quelato, na prevenção e recuperação da anemia carencial ferropriva em menores de 4 anos de idade e relacionar a evolução das crianças acompanhadas com a faixa etária, presença de diluição intrafamiliar do suplemento distribuído e quantidade de crianças menores de 5 anos existentes no núcleo familiar.

\section{MATERIAL E MÉTODO}

O trabalho foi realizado no Município de Angatuba, situado a $210 \mathrm{~km}$ da cidade de São Paulo. Selecionaramse 269 crianças com idades compreendidas entre 6 e 42 meses, matriculadas no Programa de Suplementação Alimentar da Secretaria de Estado da Saúde e da Prefeitura Municipal. A cada beneficiário foi distribuído diariamente um litro de leite in natura, fortificado com $3 \mathrm{mg}$ de ferro aminoácido quelato (ferroquel), durante 12 meses.

O leite distribuído era recolhido pelos produtores da região e entregue em cinco postos de distribuição espalhados pelo município. Ao recebê-lo, os responsáveis pelo Programa adicionavam o ferroquel antes de entregá-lo à população. Cada beneficiário retirava o leite no posto mais próximo de sua residência, nos sete dias da semana.

Antes de se iniciar a fortificação, todas as crianças selecionadas foram avaliadas do ponto de vista clínico, antropométrico e hematológico. A avaliação hematológica consistiu de dosagem de hemoglobina. Excluiu-se da 
amostra os portadores de patologias que interferissem no processo normal de crescimento e desenvolvimento, como: cardiopatias, síndromes neurológicas e outras.

O acompanhamento do programa foi feito através de avaliações trimestrais de cada beneficiário, quando repetiam-se os procedimentos iniciais. Durante essas avaliações obtinham-se informações sobre a aceitação, quantidade média ingerida/dia, presença ou não de diluição intrafamiliar do suplemento e composição familiar. Para fins de simplificação, serão apresentados apenas os resultados das avaliações hematológicas iniciais, dos 6 e dos 12 meses de intervenção.

O sangue foi coletado por punção digital e a dosagem de hemoglobina foi feita pelo método colorimétrico em aparelhos Hemocue. Para caracterização da anemia foi adotado o critério da $\mathrm{OMS}^{12}$, que estabelece o limite de $11,0 \mathrm{~g} / \mathrm{dl}$ para as idades estudadas.

Para a análise dos resultados utilizou-se:

- Teste G de Cochran ${ }^{14}$, com a finalidade de comparar as percentagens de crianças com anemia observadas nos momento anterior e após 6 e 12 meses de acompanhamento. Este teste foi aplicado separadamente para cada grupo etário, níveis de hemoglobina, presença de diluição intrafamiliar e número de menores de 5 anos existentes no núcleo familiar.

- Teste do Qui Quadrado, para comparar o decréscimo da ocorrência de anemia em famílias em que ocorria a diluição intrafamiliar do suplemento distribuído e naquelas com uma ou mais crianças menores de 5 anos.

- Partição do Qui-Quadrado, com a finalidade de comparar, por faixa etária, o número de crianças que se recuperaram da anemia a partir do momento inicial e após 6 e 12 meses de acompanhamento.

- Análise de variância para grupos não independentes $1^{6}$, com a finalidade de comparar, para cada criança, os níveis de hemoglobina observados no momento anterior e após 6 e 12 meses de intervenção. Esta análise foi aplicada, em separado, para cada um dos grupos etários. Repetiu-se o procedimento para os diferentes níveis de hemoglobina iniciais.

\section{RESULTADOS}

Antes de se iniciar a intervenção (Fig. 1), a anemia estava presente em 62,3\% das crianças. Após 6 meses de suplementação, este percentual reduziu-se a $41,8 \%$ e, ao final de um ano de uso do leite fortificado, para $26,4 \%$. O Teste de Cochran apontou redução significante da presença de anemia nas crianças acompanhadas, durante os 12 meses do estudo.

Ao relacionar a redução da ocorrência de anemia com a faixa etária dos beneficiários do programa, a Figura 2 mostrou que as maiores reduções ocorreram entre os 12 e 23 meses de idade (de $72,7 \%$ antes da intervenção para $21,6 \%$ após um ano de uso do leite fortificado), seguida dos menores de 1 ano (de

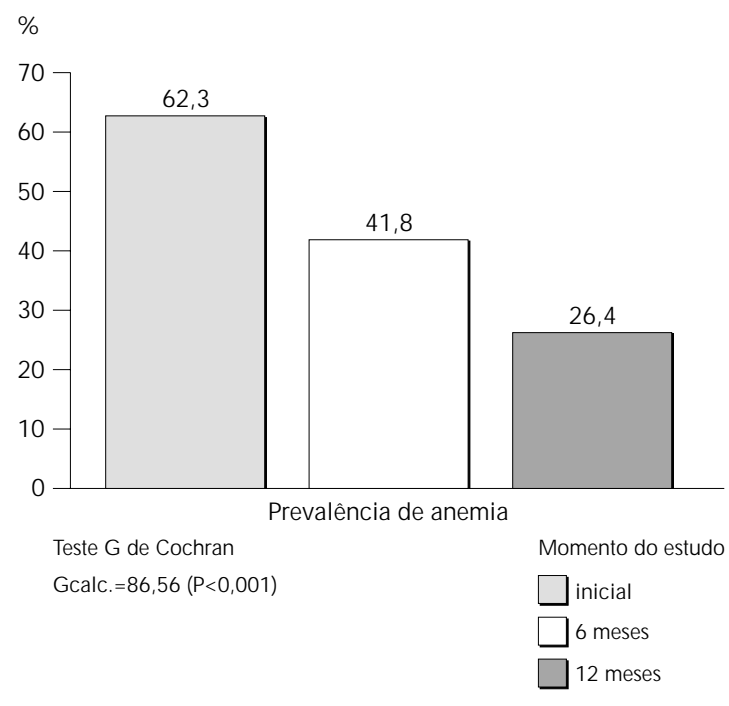

Figura 1 - Evolução dos percentuais de anemia no momento inicial e após 6 e 12 meses de uso do leite fluido fortificado em crianças menores de 4 anos do Município de Angatuba, SP.

$72,0 \%$ para $39,0 \%$ ), de 24 a 36 meses (de $44,4 \%$ para $20,0 \%$ ) e dos maiores de 36 meses (de 25,0\% para $12,5 \%$ ). O Teste de Cochran apontou reduções estatisticamente significantes nas três faixas etárias inferiores e não significantes em maiores de 36 meses de idade. A partição do Qui-Quadrado comparando, por grupo etário, o número de crianças que alcançaram níveis de hemoglobina superiores a $11,0 \mathrm{~g} / \mathrm{dl}$ apontou que as crianças de 12 a 23 meses foram as que apresentaram as melhores evoluções.

O comportamento das médias das hemoglobinas, em função da idade das crianças (Tabela 1), apontou incremento de $1,5 \mathrm{~g} / \mathrm{dl}$ na faixa dos 12 aos 23 meses; de $0,9 \mathrm{~g} / \mathrm{dl}$ em menores de um ano; de $1,0 \mathrm{~g} / \mathrm{dl}$ dos 24 aos 35 meses e de $0,3 \mathrm{~g} / \mathrm{dl}$ nos maiores de 36 meses de idade. A análise de variância dos resultados demonstrou incrementos significantes nas faixas de menores de 36 meses e não significantes naquelas com mais de 36 meses de idade.

No momento anterior à intervenção, das 239 crianças acompanhadas, $24,7 \%$ apresentavam anemia grave $\mathrm{Hb}<9,5 \mathrm{~g} / \mathrm{dl}, 37,6 \%$, anemia moderada $\mathrm{Hb}$ entre 9,5 e $10,9 \mathrm{~g} / \mathrm{dl}$, e $37,6 \%$ não apresentavam anemia. Ao final de 12 meses, 59,3\% daquelas que apresentavam anemia grave haviam atingido níveis de hemoglobina superiores a $11,0 \mathrm{~g} / \mathrm{dl}$. Entre as que apresentavam anemia moderada, $66,7 \%$ se recuperaram. No grupo de crianças sem anemia verificouse que 10,0\% desenvolveram anemia (Fig. 3). O Teste $\mathrm{G}$ de Cochan demonstrou índices de recuperação significantes para os dois grupos de crianças com ane- 
Tabela 1 - M édia das hemoglobinas e seus respectivos desvios-padrão, segundo a faixa etária, no momento anterior ( $\mathrm{Hb1}$ b e após 6 (Hb2) e 12 meses (Hb3) de uso do leite fortificado em crianças do Município de Angatuba, SP.

\begin{tabular}{|c|c|c|c|c|c|}
\hline \multirow{2}{*}{$\begin{array}{l}\text { Faixa etária } \\
\text { (meses) }\end{array}$} & \multirow{2}{*}{$\begin{array}{c}\text { Amostra } \\
\mathrm{N}\end{array}$} & \multicolumn{4}{|c|}{ M édias das Hemoglobinas e Desvios-Padrão (g/dl) } \\
\hline & & $\mathrm{Hbl}$ & $\mathrm{Hb2}$ & $\mathrm{Hb3}$ & Análise de Variância \\
\hline$<12$ & 82 & $10,2 \pm 1,3$ & $10,5 \pm 1,5$ & $11,1 \pm 1,3$ & $\begin{array}{c}\mathrm{Fc}=10,33(\mathrm{P}<0,001) \\
\mathrm{Hb} 3>\mathrm{Hb} \text { e } \mathrm{Hb} 1\end{array}$ \\
\hline $12 \geq 23$ & 88 & $10,1 \pm 1,6$ & $10,9 \pm 1,6$ & $11,6 \pm 1,1$ & $\begin{array}{c}\mathrm{FC}=51,64(\mathrm{P}<0,001) \\
\mathrm{Hb} 3>\mathrm{Hb} 2>\mathrm{Hb} 1\end{array}$ \\
\hline $24 \geq 35$ & 45 & $11,0 \pm 1,3$ & $11,7 \pm 1,2$ & $12,0 \pm 1,2$ & $\begin{array}{c}\mathrm{Fc}=17,02(\mathrm{P}<0,001) \\
\mathrm{Hb} 3 \text { e Hb2 }>\mathrm{Hb} 1\end{array}$ \\
\hline$\geq 36$ & 24 & $11,8 \pm 1,3$ & $12,2 \pm 1,1$ & $12,1 \pm 1,0$ & $\mathrm{FC}=1,29(\mathrm{NS})$ \\
\hline
\end{tabular}

mia no momento inicial, bem como, significância no aparecimento de anemia naquelas sem anemia antes da intervenção.

O incremento médio das hemoglobinas, nas crianças que apresentavam anemia grave, foi de $2,7 \mathrm{~g} /$ dl durante os 12 meses de fortificação do leite. Naquelas com anemia moderada, este incremento foi de $1,0 \mathrm{~g} / \mathrm{dl} \mathrm{e}$, nas sem anemia, a média das hemoglobinas permaneceu inalterada (Tabela 2). A análise de variância para os três grupos acompanhados revelou incrementos estatisticamente significantes nas crianças que apresentavam anemia grave e moderada, e não significantes nas que não apresentavam anemia.

Relacionou-se a presença de anemia com duas variáveis selecionadas: a presença da diluição intrafamiliar

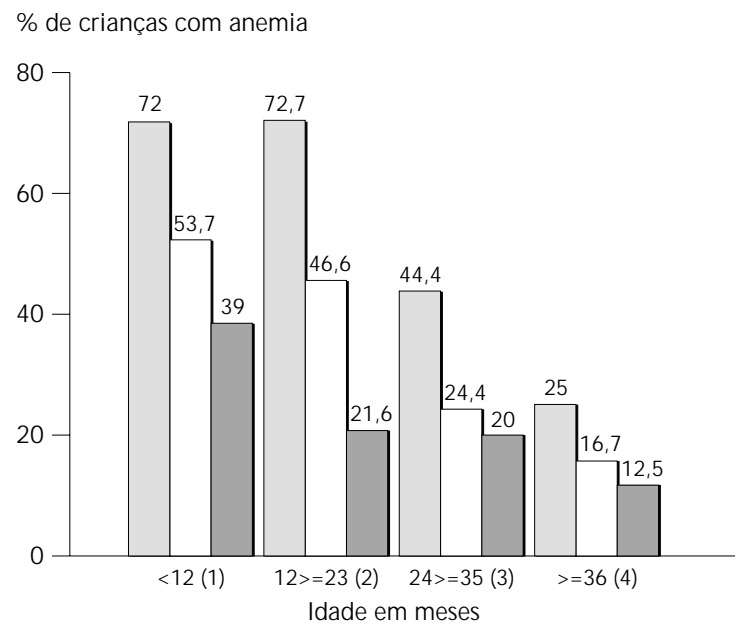

Teste $\mathrm{G}$ de Cochran

(1) G calc. $=20,33(P<0,001)$

(2) G calc. $=57,32(P<0,001)$

(3) $\mathrm{G} \mathrm{calc}=11,44(\mathrm{P}<0,01)$

(4) G calc. $=3,50$ (NS)

Momento do estudo

$\square$ anterior

$\square 6$ meses

12 meses
Figura 2 - Presença de anemia, segundo a faixa etária, no momento inicial e após 6 e 12 meses de uso do leite fluido fortificado em crianças menores de 4 anos do Município de Angatuba, SP. do suplemento distribuído e o número de crianças menores de 5 anos existentes no núcleo familiar.

A presença de diluição intrafamiliar foi detectada em $44,4 \%$ das famílias. Neste grupo, a anemia estava presente em $58,5 \%$ das crianças no início do estudo. Ao final de 12 meses, este percentual reduziu-se a $28,3 \%$. Nas famílias em que não ocorreu a diluição, a redução foi de $65,1 \%$ para $24,8 \%$ (Fig. 4). O Teste de Cochran apontou reduções significantes nos dois grupos analisados, sem que o Teste do Qui-Quadrado demonstrasse diferença entre elas.

A ocorrência de anemia decresceu de $62,7 \%$ para 19,4\% nas crianças de famílias com apenas um menor de 5 anos, durante os 12 meses do estudo (Fig. 5). Naquelas com dois ou mais menores de 5 anos, esta redução foi de $61,9 \%$ para $35,2 \%$. Os dois gru-

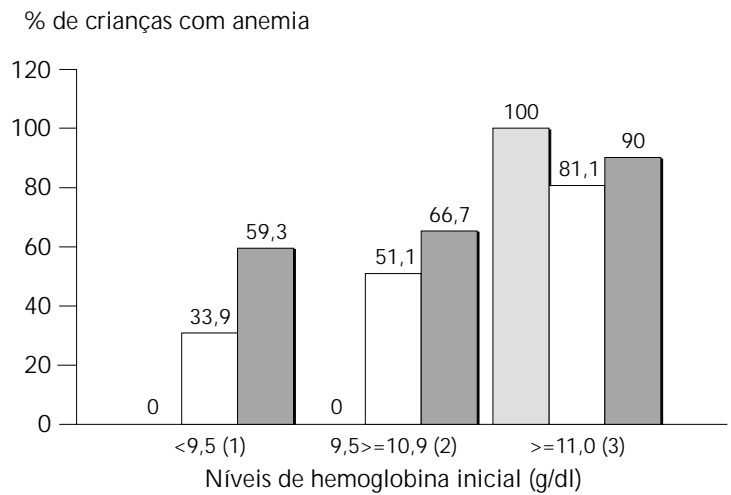

Teste $\mathrm{G}$ de Cochran

Momento do estudo

(1) G calc. $=19,63(P<0,001)$

(2) G calc. $=85,68(P<0,001)$

(3) $\mathrm{G}$ calc. $=21,70(\mathrm{P}<0,001)$ $\square$ inicial

$\square 6$ meses

12 meses

Figura 3 - Percentual de crianças sem anemia, segundo o nível de hemoglobina inicial, no momento anterior e após 6 e 12 meses de uso do leite fluido fortificado em crianças menores de 4 anos do Município de Angatuba, SP. 


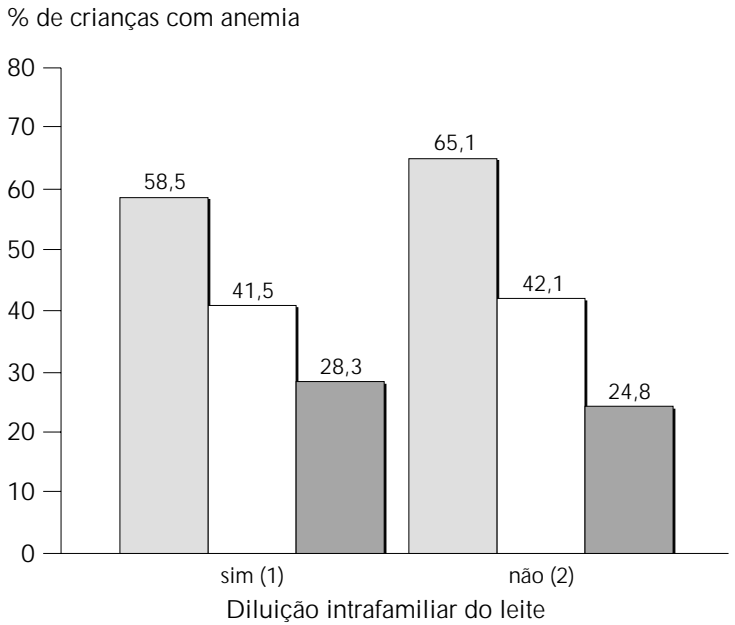

Teste $\mathrm{G}$ de Cochran

(1) G calc. $=30,88(P<0,001)$

(2) G calc. $=55,77(P<0,001)$

Q ui-Q uadrado

(1) $\times(2)$ Xcalc $=0,66$ (NS)

Figura 4 - Prevalência de anemia e presença de diluição intrafamiliar do leite fortificado, em cada um dos momentos do estudo, em crianças menores de 4 anos do Município de Angatuba, SP.
Momento do estudo

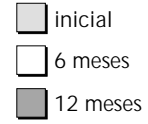

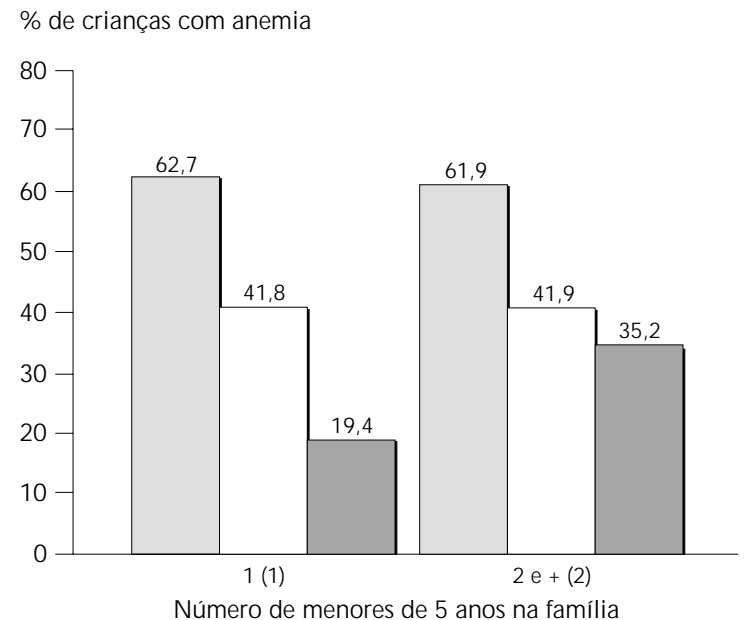

Teste $\mathrm{G}$ de Cochran

(1) G calc. $=69,15(P<0,001)$

(2) G calc. $=22,75(P<0,001)$

Q ui-Quadrado

(1) $\times(2)$ Xcalc $=4,52$ (NS)

Tabela 2 - Média das hemoglobinas e seus respectivos desvios-padrão, em função dos níveis de hemoglobina apresentados antes da intervenção ( $\mathrm{Hb}$ ) e após $6(\mathrm{Hb}$ ) e 12 meses $(\mathrm{Hb} 3)$ de uso do leite fortificado em menores de 4 anos do M unicípio de Angatuba, SP.

\begin{tabular}{|c|c|c|c|c|c|}
\hline \multirow{2}{*}{$\begin{array}{l}\mathrm{Hb} \text { inicial } \\
(\mathrm{g} / \mathrm{dl} .)\end{array}$} & \multirow{2}{*}{$\begin{array}{c}\text { Amostra } \\
\mathrm{N}\end{array}$} & \multicolumn{4}{|c|}{ M édias das Hemoglobinas e Desvios-Padrão (g/dl) } \\
\hline & & $\mathrm{Hb1}$ & $\mathrm{Hb} 2$ & $\mathrm{Hb3}$ & Análise de Variância \\
\hline$<9,5$ & 59 & $8,5 \pm 0,9$ & $9,9 \pm 1,7$ & $11,2 \pm 1,1$ & $\begin{array}{c}\mathrm{Fc}=80,47(\mathrm{P}<0,001) \\
\mathrm{Hb} 3>\mathrm{Hb} 2>\mathrm{Hb} 1\end{array}$ \\
\hline $9,5 \geq 10,9$ & 90 & $10,3 \pm 0,4$ & $11,0 \pm 1,2$ & $11,3 \pm 1,2$ & $\begin{array}{c}\mathrm{Fc}=33,34(\mathrm{P}<0,001) \\
\mathrm{Hb} 3>\mathrm{Hb}>\mathrm{Hb} 1\end{array}$ \\
\hline$\geq 11,0$ & 90 & $12,0 \pm 0,7$ & $11,9 \pm 1,3$ & $12,0 \pm 1,1$ & $\mathrm{Fc}=1,17(\mathrm{NS})$ \\
\hline
\end{tabular}

pos apresentaram reduções significantes pelo Teste de Cochran e o Teste do Qui-Quadrado não encontrou diferenças entre elas.

\section{COMENTÁRIOS E CONCLUSÕES}

A experiência com a fortificação do leite fluido com $3 \mathrm{mg}$ de ferro aminoácido quelato, por litro, foi bastante positiva. Tratando-se de um experimento inédito, alguns pontos merecem consideração:

\section{Do método}

- O ferro aminoácido quelato apresentou boa solubilidade.

- A quantidade de ferroquel utilizada na fortificação de um litro de leite não alterou a cor e o sabor do produto final.
- A adição de $3 \mathrm{mg}$ de ferroquel por litro de leite decorreu do fato de o produto ter apresentado, em ratos, absorção quase 4 vezes superior a do sulfato ferroso ${ }^{4}$. A ligação do íon ferro com o aminoácido possibilitaria sua absorção de forma passiva, sem interferência de agentes inibidores presentes no lúmen intestinal. Entretanto, como a literatura especializada não registra nenhum experimento clínico que comprove efetivamente esta informação, a quantidade utilizada foi calculada levando-se em consideração trabalho anterior ${ }^{21}$, que obteve excelentes índices de redução de anemia, em crianças suplementadas diariamente com $6 \mathrm{mg}$ de ferro elementar na forma de sulfato ferroso. Estimou-se que o ferroquel apresenta absorção duas vezes superior à do sulfato ferroso e 
optou-se pela fortificação do leite com $3 \mathrm{mg}$ de ferroquel por litro.

- A utilização do leite in natura, na alimentação de menores de 2 anos, é prática comum no Interior do Estado de São Paulo. Na maioria das regiões, o mercado oferece o leite pasteurizado, mas há tendência para o consumo do leite in natura devido à divulgação do conceito de que "o leite puro da fazenda é melhor para a saúde que o de saquinho (pasteurizado), além de ser mais barato". Entretanto, o acompanhamento da qualidade do leite in natura tem mostrado, em análises microscópicas, presença de fragmentos de insetos, pus, sangue, pelos de animais e de humanos e fezes de insetos. Análises microbiológicas demonstraram número de coliformes muito acima do permitido pela lei, resultando num produto impróprio para o consumo humano. Em função desses resultados, a Prefeitura Municipal de Angatuba adquiriu uma miniusina para pasteurização do leite distribuído.

- Durante o experimento, observou-se que, na fervura, grande parte do ferro (aproximadamente $70 \%$ ) fixava-se na nata e que, culturalmente, as mães não têm o hábito de utilizá-la na alimentação de seus filhos. A partir dessas constatações, foi necessário fazer trabalho educativo para informar e enfatizar a necessidade das mães administrarem a nata às crianças-alvo. Na pasteurização, o leite sofre um processo de homogeneização, no qual a gordura é reduzida a micropartículas e emulsificada, reduzindo a $8 \%$ a perda do ferro que migra para a nata.

\section{Dos Resultados}

- Após um ano de fortificação do leite fluido com $3 \mathrm{mg}$ de ferroquel/litro, a redução do percentual de crianças com anemia foi bastante significativa.

- No grupo de menores de um ano foram registradas as menores reduções, constatando-se que a quantidade suplementar de $3 \mathrm{mg} /$ dia de ferroquel foi insuficiente para suprir as necessidades do íon ferro dessa faixa etária. Estas crianças apresentavam algumas características que as diferenciavam dos demais grupos etários acompanhados, a saber: elevada velocidade de crescimento, maior necessidade de íon ferro/ dia, cultura alimentar equivocada com dieta pouco diversificada, na qual o leite se apresenta como o principal, senão o único alimento ofe- recido. A partir desta constatação levantou-se a hipótese de que a quantidade de $3 \mathrm{mg}$ de ferroquel por dia seria suficiente caso sejam efetivadas medidas preventivas objetivando a redução do número de nascimentos prematuros e de peso insuficiente para a idade gestacional, o abandono precoce do aleitamento materno, a educação nutricional para reforçar a preconização de uma alimentação mais diversificada como complementação ao aleitamento materno, entre outras. Desta forma o suplemento férrico oferecido estaria atuando no seu real papel, como profilático da carência deste mineral.

- As crianças com anemia grave incorporaram o íon ferro com maior velocidade do que aquelas com anemia moderada. O incremento da média de hemoglobina observada durante o ano de acompanhamento reforça esta hipótese e indica que quanto maior a carência, maior a absorção do ferro suplementar oferecido. Os elevados índices de recuperação da anemia encontrados tanto nas crianças com anemia grave, como moderada, demonstram a eficácia deste tipo de intervenção em populações de risco.

- Constatou-se o aparecimento de anemia em $10 \%$ das crianças não-anêmicas, no início do estudo. Uma das maiores limitações do trabalho foi a utilização apenas da hemoglobina como aferidor das condições hematológicas das crianças acompanhadas. Por se tratar de uma pesquisa operacional, não houve possibilidade de realizar testes laboratoriais que avaliassem as reservas de ferro no organismo. A dosagem de ferritina sérica poderia explicar este fato, ou seja, que neste grupo de crianças as baixas reservas de ferro, acompanhadas de algum fator desencadeante (infecções, infestações, por exemplo), foram os responsáveis pelo aparecimento de anemia, apesar da suplementação férrica administrada.

- A diluição intrafamiliar do suplemento distribuído é uma questão que preocupa os técnicos responsáveis pelo planejamento, execução e avaliação dos Programas de Suplementação Alimentar. Esta informação obtida em entrevista pode ser passível de erro. As mães temem a suspensão do suplemento alimentar, caso fosse constatado que o leite não é alimento exclusivo da criança-alvo. No presente estudo, esta questão foi verificada com o número de menores de 5 anos existentes no núcleo familiar - informação mais confiável. Partiu-se da hipótese que a presença de outra criança na família facilita- 
ria a partilha do alimento distribuído. Os resultados obtidos demonstram que, tanto a presença da diluição intrafamiliar, como o número de crianças menores de 5 anos, não foram um fator limitante na recuperação da anemia carencial ferropriva, apesar de comprovado que a redução foi significativamente superior em famílias com apenas uma criança menor de 5 anos.

- Ao comparar os resultados obtidos no presente estudo, com os encontrados com a fortificação do leite em pó com sulfato ferroso e vitaminas (A, C e D), pode-se constatar que o decréscimo de crianças anêmicas foi mais acentuado quando utilizado o leite em pó. Uma ingestão média diária de $6 \mathrm{mg}$ de íon ferro, sob a forma de sulfato ferroso, foi capaz de reduzir a menos de $20 \%$ o percentual de crianças que permaneciam anêmicas após 6 meses de suplementação ${ }^{21}$, enquanto, no presente estudo, o percentual encontrado no mesmo tempo de seguimento foi de $41,8 \%$. Seria lógico pensar que o leite fluido, devido à sua durabilidade e facilidade de manipulação, facilitaria a prática da diluição intrafamiliar. Por outro lado, o leite em pó seria mais dirigido à criança-alvo, em decorrência da cultura alimentar existente: "o leite em pó é para ser dado a crianças pequenas". Entretanto, ao analisar os resultados obtidos em famílias com apenas uma criança menor de 5 anos, em que, certamente, eram insignificantes as possibilidades de ocorrer a diluição intrafamiliar do suplemento distribuído, verificou-se que, ainda assim, a velocidade de decréscimo da ocorrência de anemia era menor que a encontrada com o uso do leite em pó.

- Como o leite em pó foi enriquecido com ferro e vitaminas A, C e D, poder-se-ia imaginar que o acréscimo das vitaminas principalmente as

\section{REFERÊNCIAS BIBLIOGRÁFICAS}

1. AMERICAN ACADEMY OF PEDIATRICS. Committee on Nutrition. Iron supplementation for infants. Pediatrics, 58: $765-8,1976$.

2. BATISTA FILHO, M. \& BARBOSA, N.P. Pró-Memória: alimentação e nutrição no Brasil: 1974-1984. Brasília, INAN, 1985.

3. COOK, J.D. \& REUSSER, M.E. Iron fortification: an update. Am. J. Clin. Nutr., 38: 459-68,1983.

4. CRISTENSEN, J.M. Effects of divalent amino acids on iron absorption. J. Pharm. Sci., 73: 1245-7, 1984. vitaminas A e C estariam contribuindo para um retorno mais rápido à situação de adequação dos níveis de hemoglobina. Estudo recente relacionou o papel da vitamina A na absorção, transporte e armazenamento do ferro e síntese da hemoglobina ${ }^{23}$.

- A afinidade do íon ferro com a gordura do leite foi comprovada no presente estudo. A utilização do leite in natura pode ter sido um fator muito importante na explicação das diferenças encontradas com o uso do leite em pó. As mães habitualmente não utilizam, na alimentação de seus filhos, a nata formada na fervura do leite. De fato, as reduções nos percentuais de anemia aumentaram sensivelmente após o trabalho educativo.

- O ferroquel, dependendo da quantidade acrescentada ao leite, apresenta vantagens: não precipita e não altera cor e sabor, características estas não alcançadas com o sulfato ferroso.

As hipóteses discutidas abrem caminho para outras investigações e confirmam a política de fortificação dos alimentos como uma das principais estratégias na prevenção e controle da carência de ferro em populações com elevadas prevalências de anemia. Reforçam, ainda, a exemplo de outro estudo ${ }^{21}$, que pequenas doses suplementares de ferro/dia são suficientes, em um espaço de tempo relativamente curto, para controlar esta deficiência.

\section{AGRADECIMENTOS}

À população de Angatuba, ao Prefeito Municipal, José Emílio Carlos Lisboa, e à Coordenadora Municipal de Saúde, Abigail de Almeida Lisboa, pelo apoio e pioneirismo demonstrados. À Maria dos Anjos Carriel Ferreira e Elenira Maria Ramos Nunes, pela empenho e dedicação na coordenação do trabalho no município.

5. DeMAYER, E.M. Preventing and controlling iron deficiency anaemia through primary care. Geneva, World Health Organization, 1989.

6. INTERNATION CONFERENCE ON NUTRITION, Rome, 1992. Final report. Rome, FAO/WHO, 1992.

7. JOÃO,W.S.J. Prevalência de anemia na população atendida nos postos de saúde da Secretaria de Saúde do Estado do Pará e em escolares participantes do Programa Nacional de Alimentação Escolar: relatório anual. Belém, Convênio INAN/UFPA, 1983. 
8. MacPHAIL, A.P. \& BOTHWELL,T.H. Fortification of the diet as a strategy for preventing iron deficiency. Acta Paediatr. Scand., 361: (suppl.) 114-24,1989.

9. MONTEIRO, C.A. Saúde e nutrição das crianças de São Paulo: diagnóstico, contrastes sociais e tendências. São Paulo, Hucitec / ED. USP, 1988.

10. NESTLÉ NUTRITION FOUNDATION. El hierro en la alimentación del lactante y del niño. Nestlé Nutrition, 1984.

11. OLIVARES, M.; WALTER, T.; HERTRAMPF, E.; PIZARRO, F.; STEKEL, A. Prevention of iron deficiency by milk fortification. Acta Paediatr. Scand., 361: (suppl.) 109-3, 1989.

12. ORGANIZACION MUNDIAL DE LA SALUD. Lucha contra la anemia nutricional, especialmente contra la carencia de hierro. Ginebra, 1975. (OMS - Serie de Informes Tecnicos, 580).

13. ROMANI, S.A.M.; LIRA, P.I.C.; BATISTA FILHO, M.; SEQUEIRA, L.A.S.; FREITAS, C.L.C. Anemias em préescolares: diagnóstico, tratamento e avaliação, Recife-Pe, Brasil. Arch. Latinoam. Nutr., 67: 159-67, 1991.

14. SIEGEL, S. Estadistica no parametrica. Mexico, Ed. Trillas, 1975.

15. SIGULEM, D.M.; TUDISCO, E.S.; GOLDENBERG, P.; ATHAIDE, M.M.M.; VAIMAN, E. Anemia ferropriva em crianças no Município de São Paulo. Rev. Saúde Pública, 12: 168-78, 1978.

16. SOKAL, R.R. \& ROHLF, F.J. Biometry. San Francisco, W.H. Freeman and Company, 1969.
17. STEKEL, A.; OLIVARES, M.; CAYAZZO, M.; CHADUD, P.; LLAGUNO, S.; PIZARRO, F. Prevention of iron deficiency by milk fortification. II - A field trial with a fullfat acidified milk. Am. J. Clin. Nutr., 47: 265-9, 1988.

18. TORRES,M.A.A. Estado nutricional e aspectos sócioeconômicos de famílias rurais do Trópico Semi-árido (Nordeste do Brasil). Recife,1982. [Dissertação de Mestrado- Universidade Federal de Pernambuco].

19. TORRES, M.A.A.; SATO, K.; SOUZA QUEIROZ, S. Anemia em crianças menores de dois anos atendidas nas unidades básicas de saúde no Estado de São Paulo, Brasil Rev. Saúde Pública, 28 :290-4, 1994.

20. TORRES, M.A.A.; SATO, K.; SOUZA QUEIROZ, S. Terapêutica com doses profiláticas de sulfato ferroso, como medida de intervenção no combate à carência de ferro, em crianças atendidas em unidades básicas de saúde. Rev. Saúde Pública, 28 : 410-5, 1994.

21. TORRES, M.A.A.; SATO, K.; SOUZA QUEIROZ, S. Efeito do uso do leite fortificado com ferro e vitamina $\mathrm{C}$ sobre os níveis de hemoglobina e condição nutricional de crianças menores de dois anos. Rev. Saúde Pública, 29: 301-7, 1995.

22. TURCONI, S.J. \& TURCONI, V.L. Anemia ferropriva: incidência em uma população infantil. Pediatr. Mod., 27: (2), 1992 .

23. WOLDE-GEBRIEL, Z.; WEST, C.E.; GEBRU, H.; TADESSE, A.S.; FISSEHA, T.; GABRE, P.; ABOYE, C.; AYANA, G.; HAUTVAST, J.G.A.J. Interrelationship between vitamin A, iodine, and iron status in schoolchildren in Shoa region, Central Ethiopia. Br. J. Nutr., 70: 593-607, 1993. 